\title{
Meta-análisis
}

Pensar en Movimiento:

Revista de Ciencias del Ejercicio y la Salud

ISSN 1659-4436

Vol. 8, No. 1, pp. 9- 19

\section{META-ANÁLISIS SOBRE EL EFECTO DEL EJERCICIO ACUÁTICO EN LA SINTOMATOLOGÍA DE LA FIBROMIALGIA}

\author{
Diana Villalobos Blanco, M.Sc. ${ }^{1,2(A, C, D, E)}$ y Pedro Carazo Vargas, M.Sc. ${ }^{3(B, C, D, E)}$ \\ e-mail: pedro.carazo@ucr.ac.cr \\ ${ }^{1}$ Escuela de Nutrición, Universidad de Costa Rica \\ ${ }^{2}$ Escuela de Ciencias del Deporte, Universidad Nacional \\ ${ }^{3}$ Escuela de Educación Física y Deportes, Universidad de Costa Rica
}

Manuscrito recibido: 13/07/2010; aceptado: 27/09/2010

\begin{abstract}
RESUMEN
Villalobos-Blanco, D., y Carazo-Vargas, P. (2010). Meta-análisis sobre el efecto del ejercicio acuático en la sintomatología de la fibromialgia. PENSAR EN MOVIMIENTO: Revista de Ciencias del Ejercicio y la Salud, 8 (1), 9-19. La sintomatología de la fibromialgia (FM) genera alteraciones en la calidad de vida de las personas que la padecen, por ello se ha estudiado el efecto de diferentes terapias para aliviar estos síntomas. El ejercicio acuático ha mostrado resultados positivos en muchos estudios, por lo cual se estableció el objetivo de analizar metaanalíticamente las características de este tipo de ejercicio en los síntomas de la enfermedad. Se incluyeron un total de 16 estudios (únicamente estudios experimentales puros publicados entre los años 1998 y 2008) para un total de 196 tamaños de efecto. Se realizó un primer análisis comparando el tipo de actividad física realizada y las variables moderadoras incluyendo únicamente estudios con actividades acuáticas. Al analizar el tipo de actividad realizada, la única que mostró diferencias significativas respecto a la no realización de ejercicio fue la ejecutada en un ambiente acuático. En este tipo de actividad se hallaron tamaños de efecto significativos para todas las edades estudiadas, los años de padecer la enfermedad, la frecuencia del ejercicio, las intensidades, el tamaño de grupo, las sesiones totales, los síntomas y componentes de aptitud física. En general, se recomienda realizar la actividad fomentando los grupos grandes, que favorezcan los lazos sociales, aplicando una frecuencia de tres sesiones semanales, con una duración de 60 minutos, indistintamente de la intensidad.
\end{abstract}

PALABRAS CLAVE: Fibromialgia, tamaño del efecto, ejercicio acuático, meta-análisis. 


\begin{abstract}
Villalobos-Blanco, D., \& Carazo-Vargas, P. (2010). Effect of aquatic exercise on fibromyalgia symptoms: a meta-analysis. PENSAR EN MOVIMIENTO: Revista de Ciencias del Ejercicio y la Salud, 8(1), 9-19. Fibromyalgia (FM) symptoms cause a decline in the quality of life of people who suffer it, ranging from mild to severe; for that reason diverse therapies have been studied to lessen the health consequences of this syndrome. Aquatic exercises have shown positive results in many studies, setting the objective of performing a meta-analysis of the characteristics of this type of exercise and its association with symptoms caused by fibromyalgia. Sixteen studies were included (only pure experimental studies published between 1998 and 2008), from which a total of 196 effect sizes were calculated. An initial analysis compared the type of physical activity and then analyzed the moderating variables including studies that used only water activities. When analyzing the type of activity performed, the only one showing significant differences when compared to the control group was the one executed in an aquatic environment. From this subset, significant effect sizes were found at all ages studied, years of suffering the disease, exercise frequency, intensity, size of group, number of sessions, symptoms and all the components of physical fitness analyzed. In general, the activity is recommended to be performed in large groups, encouraging social ties, with a frequency of three sessions of 60 minutes per week, regardless of the intensity.
\end{abstract}

KEY WORDS: Fibromyalgia, effect size, aquatic exercises, meta-analysis.

El Colegio Americano de Reumatología publicó en 1990 por primera vez los criterios de clasificación de la fibromialgia. Estos criterios de diagnóstico han permitido a diversos investigadores alrededor del mundo estudiar la expresión de los síntomas de la enfermedad, llegando a la conclusión de que los sujetos estudiados comparten la presencia de dolor crónico generalizado y la evidencia de alteraciones en el procesamiento del dolor, lo cual se comprueba al contabilizar los puntos sensibles (Clauw \& Crofford, 2003). Se determina que una persona padece fibromialgia cuando ha presentado dolor generalizado por más de 3 meses en al menos 11 de los 18 puntos sensibles. Estas zonas se ubican en brazos, glúteos, pecho, rodillas, espalda baja, cuello, caja torácica, hombros y muslos (Brecher \& Cymet, 2004).

El síndrome de la fibromialgia (FM) se ha encontrado en todos los grupos étnicos estudiados hasta la fecha. La enfermedad no se limita a naciones industrializadas y opulentas. Con una prevalencia que va del 2 al 12\% de la población en general, ésta debe ser observada como una condición médica común. Su presencia se incrementa con la edad y de manera más dramática en mujeres con un máximo en la edad correspondiente a la quinta y sétima década (Wolfe, Ross, Anderson, Russell, \& Hebert, 1995).

Las principales quejas presentadas por estas personas, son el dolor en varios sitios del cuerpo, fatiga y complicaciones del sueño. Los pacientes usualmente se quejan de dolor de espalda, el cual puede irradiar a los glúteos y las piernas. También, algunos sujetos presentan rigidez en el cuello y hombros. Esta rigidez usualmente se presenta en las mañanas y mejora en el transcurso del día. Así mismo, se experimenta cansancio y fatiga, inclusive al caminar. Muchos pacientes se despiertan con frecuencia en la noche y tienen dificultad para volver a conciliar el sueño. Aunque no se dé inflamación en las articulaciones, sí se percibe la presencia de ésta. Igualmente, muchas personas con esta enfermedad presentan problemas cognitivos como pérdida de memoria, ausencia de palabras y vocabulario escaso. Otras manifestaciones observadas son mareos, ansiedad y depresión. Los síntomas se pueden agravar con el clima húmedo y frío, la falta de sueño y el estrés, entre otros (Chakrabarty \& Zoorob, 2007).

Actualmente se desconoce la causa de esta enfermedad y por ende, esto es origen de debate. Probablemente se trate de un proceso multifactorial. 
Se ha considerado que pudiera ser un trastorno muscular primario, un proceso de tipo psicosomático o una disfunción neuroendrocrina. Existen diversos factores que pueden estar involucrados en el desarrollo de la enfermedad, entre ellos: aspectos genéticos, alteraciones neurofisiológicas o de neurotransmisores, trastornos psiquiátricos como la depresión y la ansiedad, así como factores sociales y ambientales (Rosas, 2006).

Aparte de las anormalidades sensoriales y bioquímicas, la contribución de factores psicológicos al dolor de la FM también debe de tomarse en cuenta. Los pacientes con FM son más susceptibles que la población en general a ser diagnosticados con desordenes psiquiátricos, particularmente con depresión y ansiedad (McBeth, Macfarlane, Benjamin \& Silman, 2001). Existe evidencia que sugiere que los problemas psiquiátricos ocurren en mayor proporción en pacientes con FM en comparación con otros pacientes que padecen de dolor crónico (Hassett, Cone, Patella \& Sigal, 2000).

Teniendo en cuenta que no existe ningún fármaco capaz de controlar la enfermedad completamente (Rosas, 2006), el ejercicio, al poder contar con propiedades analgésicas, ha representado una de las áreas más estudiadas en el tratamiento de la FM. Diversos tipos de ejercicio pueden ser tolerados por estos pacientes, en especial el ejercicio aeróbico (Busch, Schachter, Peloso \& Bombardier, 2002 y Rooks, Silverman \& Kantrowitz, 2002).

Los mecanismos responsables del efecto analgésico del ejercicio todavía no son claros. Una hipótesis propone que la activación del sistema opioide endógeno, por su capacidad de aplacar los estímulos dolorosos, es responsable del efecto analgésico, sin embargo, pueden influir también mecanismos no opioides mediados por otras sustancias como la hormona del crecimiento y la corticotropina. Así mismo, el ejercicio puede mejorar el bienestar de los pacientes al prevenir la hipoxia muscular en los pacientes fibromiálgicos. Otro efecto positivo es el restablecimiento en el sueño. Los desórdenes del sueño juegan un rol importante en la etiología de la FM, por lo que una mejora en éste puede contribuir a alivianar otros síntomas (Altan, Bingöl, Aykaç, Koç \& Yurtkuran, 2004).

El ejercicio también puede producir un efecto positivo en algunos factores psicológicos como la ansiedad y la depresión, y además, mejora la sensación de bienestar general y brinda un sentido de realización (Gowans, DeHueck \& Abbey, 2002).

Desde la década de los ochentas se ha estudiado el efecto del ejercicio en esta enfermedad y en años recientes se han evaluado programas de ejercicio con diferentes intensidades, distintos tipos de ejercicio o en combinaciones de éste con otras terapias no farmacológicas.

Los programas de ejercicio utilizados en personas con FM han sido de diversos tipos: aeróbico, de fuerza, flexibilidad y en piscina. Aunque los programas varían, la mayoría tienen elementos comunes: aumento gradual de la intensidad, énfasis en la importancia del ejercicio habitual y el empleo de intensidad moderada.

Las intervenciones más utilizadas han sido de ejercicio aeróbico. Estos programas, en especial los de intensidades bajas a moderadas, causan efectos positivos en cuanto a calidad de vida, el impacto de la enfermedad y el dolor. Sin embargo, algunos estudios han mostrado algunos inconvenientes, como falta de adherencia al programa, exacerbación del dolor, estrés e invalidez, entre otros. (Peterson, 2007).

Como respuesta a esta situación, el ejercicio acuático puede traer beneficios extra debido a los cambios en las propiedades biofísicas que facilitan la técnica como: la flotabilidad, el gradiente de la presión hidrostática, la viscosidad, la temperatura del agua. La estimulación termal, táctil y la resistencia, pueden llevar a una mayor relajación, reducción de la sobrecarga articular, vasodilatación por calentamiento y analgesia. Cuando el cuerpo se mueve debajo del agua causa turbulencia; esta situación genera una fuerza hidrodinámica que provoca resistencia a los movimientos corporales. Así mismo, un gran repertorio de movimiento es posible, permitiendo propiocepción, balance corporal, fuerza, y ejercicios aeróbicos (Assis et al., 2006). En general, el ejercicio acuático mejora la flexibilidad y la fuerza en un ambiente que reduce el malestar causado por la FM. El agua ayuda a dar soporte al cuerpo y amortigua la carga sobre los músculos y las articulaciones. El hecho de caminar en el agua provee un ejercicio que no causa estrés mecánico al cuerpo. Para algunos sujetos con FM, eliminar completamente el impacto del ejercicio es necesario y el efecto de flotación del agua es un ambiente idóneo.

El ejercicio en agua o hidrocinesioterapia ha demostrado su eficacia en la mejoría de síntomas 
similares a los presentados por las personas que presentan fibromialgia. En personas con osteoartritis la aplicación de un programa de ejercicios acuáticos ha mostrado resultados favorables en cuanto a la disminución del dolor y en el aumento de la calidad de vida, la fuerza, la movilidad en las articulaciones $y$ en la funcionalidad en general (Minor, Hewett, Webel, Anderson \& Kay, 1989; Hinman, Heywood \& Day, 2007; Wang, Belza, Thompson, Whitney \& Bennett, 2007).

En pacientes con FM, se han realizado estudios que han comparado el ejercicio en piscina y la no realización de ejercicio, los cuales aunque utilizando diferentes metodologías, en general coinciden en el beneficio experimentado por los individuos al participar en esta clase de programas (Gowans, deHueck, Voss \& Richardson, 1999; Gowans et al., 2001; Gusi, Tomas-Carus, Häkkinen, Häkkinen \& Ortega-Alonso, 2006; Mannerkorpi, Nyberg, Ahlmén \& Ekdahl, 2000). Entre los principales beneficios de este tipo de ejercicio se encuentra la mejoría en la capacidad cardiorrespiratoria (Gowans et al., 1999; Gowans et al., 2001 y Mannerkorpi et al., 2000), aumento de la funcionalidad, fuerza muscular, disminución del dolor, depresión y ansiedad (Gusi et al., 2006; Mannerkorpi et al., 2000) y auto-eficacia (Gowans et al., 2001).

Así mismo, se ha comparado el ejercicio acuático y el ejercicio terrestre (Jentoft, Kvalvik, \& Mengshoel, 2001; Assis et al., 2006; De Melo, de Carvalho \& do Prado, 2006), comprobando que el ejercicio acuático es equivalente, en ciertos a aspectos, al ejercicio terrestre. Sin embargo, el primero ha mostrado tener mayores mejorías en aspectos emocionales (Assis et al., 2006; Jentoft et al., 2001) y el sueño (De Melo et al., 2006).

En general los programas de ejercicio acuático han mostrado mejorías en el umbral del dolor, en la cantidad de puntos sensibles, en la calidad del sueño, en los niveles de condición física, en la calidad de vida y la función cognitiva de personas con FM. (Munguía-Izquierdo \& Legaz-Arrese, 2007). Sin embargo, estos estudios han sido dirigidos a diversas poblaciones y las características de administración del ejercicio han diferido entre sí.

El presente meta-análisis se propone inicialmente comprobar la efectividad de los programas de ejercicio en agua en el tratamiento de la fibromialgia, para posteriormente identificar las características más efectivas para reducir los síntomas, e incrementar la capacidad cardiorrespiratoria y la resistencia muscular.

\section{METODOLOGÍA}

Búsqueda de literatura. La búsqueda se realizó en las siguientes bases de datos: Embase, Ovid, Access Science, Alt-Healthwatch, Cochrane Library, EBSCOhost, Science Direct, NLM Gateway, ISI Web of Knowledge, Access Medicine y PubMed. Se utilizaron las siguientes palabras clave para la revisión: fibromyalgia, pool exercise, aquatic exercise, water exercise y swimming.

Además, se realizó una búsqueda en las siguientes revistas: Journal of Rheumatology, Rheumatology International, Medicine and Science in Sport and Exercise, Arthritis and Rheumatism Journal, Arthritis Care and Research, International Journal of Rheumatic Diseases, Clinical Rehabilitation, Clinical Journal of Pain, entre otras.

Los estudios que se incluyeron se limitaron a reportes completos de ensayos clínicos aleatorizados que examinaran la efectividad de un programa de ejercicios en agua en comparación a un grupo control o a otras intervenciones, tales como el ejercicio en piso, balneoterapia, entre otros, en adultos con FM. Así mismo, sólo se incluyeron estudios experimentales puros para asegurar una óptima calidad y para evitar algún sesgo en los resultados. Los estudios encontrados se publicaron entre los años 1998 y 2008. La búsqueda finalizó en mayo del 2009.

Cálculo de los tamaños de efecto (ES). Siguiendo los procedimientos descritos por Thomas y French (1986), se analizaron los ES que comparan los cambios entre el pre test y el post test en los grupos experimentales y control. Para no sobreestimar los ES según el tamaño de la muestra dentro de cada estudio, se calculó la fórmula de corrección del error según sesgo y peso. Luego de agrupar los tamaños de efecto según las variables de interés, se determinó el peso promedio del ES del grupo, el error estándar y una prueba $\mathrm{Z}$ para cada grupo según las fórmulas propuestas por Thomas \& French (1986). Finalmente, se determinaron las diferencias entre los grupos mediante el empleo de un análisis de varianza.

Codificación de variables moderadoras. Con el objetivo de establecer los factores que intervienen 
en la magnitud de los efectos del ejercicio acuático en el tratamiento de la FM, se establecieron una serie de variables moderadoras que se incluyen en dos apartados. El primero incluye características de la muestra (edad y años desde el diagnóstico de la FM) y el segundo contempla las características del programa de ejercicio (tipo de actividad, intensidad, frecuencia de sesiones, duración de la sesión, total de sesiones, número de personas en el grupo, sintomatología y aptitud física.

Análisis Estadístico. Se usó el Statistical Package for the Social Sciences (SPSS) versión 10.0. Para determinar la influencia sobre el ES de cada una de las variables independientes, se empleó un análisis de varianza (ANOVA) de una vía según correspondiese y la prueba Post Hoc de Tukey.

Se utilizó la técnica de la z meta-analítica para determinar si el tamaño de efecto era estadísticamente diferente de cero, para lo cual se siguieron los procedimientos descritos por Thomas y French (1986).

Según Thomas, Salazar \& Landers (1991), se estimó la magnitud de los ES de la siguiente manera: ES < 0.41, pequeño; ES: 0.41 a 0.70 , moderado; ES > 0.70, grande.
Para la comparación entre los distintos tratamientos de la enfermedad, más específicamente ejercicio acuático, combinación de ejercicio acuático y terrestre, solamente terrestre, terapia conductual-cognitiva, balneoterapia y control, se analizaron un total de 16 estudios (196 ES). Para las otras categorías de variables moderadoras, se usaron los estudios cuyo programa de ejercicios fuera únicamente de hidrocinesioterapia (ejercicio en agua, piscina o mar) con un total de 89 ES.

En la tabla $\mathrm{N}^{\circ} 1$ se muestran los valores obtenidos al comparar las diferentes actividades realizadas por las personas que padecían FM. Mediante el análisis de varianza de una vía para grupos independientes, se analizó el efecto del tipo de tratamiento sobre la sintomatología y componentes de aptitud física, determinando que existen diferencias significativas $(F=7.35$; $\mathrm{p}<0.0001)$. Luego, el análisis post hoc de Tukey demostró que las diferencias se encontraban únicamente entre los grupos que realizaban ejercicio acuático y el grupo control. El análisis de la z metaanalítica demostró que los ES en todos los tipos de actividad eran estadísticamente diferentes de cero (ver tabla $\mathrm{N}^{\mathrm{o}} 1$ ). Además, se encontró diferencia significativa entre el ES de ejercicio acuático y el de grupo control.

\section{RESULTADOS}

Tabla $N^{0}$ 1. Análisis del tamaño del efecto (ES), según actividad física realizada

\begin{tabular}{llllll}
\hline & ES & DE & n & F & Sig. \\
\hline Tipo de actividad & & & & 7.35 & $0.0001^{*}$ \\
a. $\quad$ Ejercicio acuático ${ }^{\text {a-f }}$ & 1.05 & 1.42 & 89 & & \\
b. Ejercicio acuático y terrestre & 0.71 & 0.95 & 20 & & \\
c. Ejercicio terrestre & 0.52 & 0.37 & 14 & & \\
d. $\quad$ Terapia conductual/cognitiva & 0.33 & 0.25 & 10 & & \\
e. Balneoterapia & 0.89 & 0.99 & 10 & & \\
f. Grupo control $\quad-0.05$ & 1.13 & 51 & & \\
* Significativo con $p<0.05$ & & & & &
\end{tabular}

El superíndice ${ }^{a-f}$ indica entre cuáles ítems se encontró una diferencia estadística en el análisis post hoc.

En las tablas $\mathrm{N}^{\circ} 2$ y $\mathrm{N}^{\circ} 3$, se aprecian los valores del ES promedio y DE promedio, cantidad de ES y diferencias encontradas al analizar las características de la muestra y del ejercicio.

En éste se observa que todas las categorías de las variables moderadoras tuvieron una mejoría $(\mathrm{p}<0,05)$ en la sintomatología y componentes de aptitud física de la FM. Al analizar los ES de cada una de las características de la muestra (tabla $\mathrm{N}^{\circ} 2$ ) y del ejercicio ( $\mathrm{N}^{\circ}$ 3) mediante la z meta-analítica, se determinó que los ES en todas esas categorías eran estadísticamente distintos a cero. Además se encontró diferencia significativa entre el ES de 11 a 20 años de diagnóstico de FM y el de la categoría de “no reportado" (tabla $\mathrm{N}^{0}$ 2). En las características del ejercicio (tabla $\mathrm{N}^{0} 3$ ), se encontraron diferencias significativas entre el ES de 1 vez por semana y el de 3 veces por semana; entre 30 a 35 minutos y 60 
minutos; entre de 11 a 30 sesiones y de 31 a 50 y 21 a 30 personas.

entre grupos de menos de 10 personas y grupos de

Tabla No 2 Análisis del tamaño del efecto (ES), según características de la muestra

\begin{tabular}{lccccc}
\hline & ES & DE & n & F & Sig. \\
\hline Edad & & & & 1.62 & 0.207 \\
a. $\quad 40$ a 50 años & 1.15 & 1.62 & 65 & & \\
b. Más de 51 años & 0.73 & 0.48 & 24 & & \\
Años diagnóstico & & & & 6.60 & $0.0001^{*}$ \\
a. Menor de 10 años & 0.45 & 0.45 & 12 & & \\
b. De 11 a 20 años b-d & 0.27 & 0.27 & 15 & & \\
c. Más de 20 años & 0.73 & 0.73 & 24 & & \\
d. No reportado & 1.73 & 1.73 & 38 & & \\
\hline
\end{tabular}

* Significativo con $p<0.05$.

El superíndice ${ }^{b-d}$ indica entre cuáles ítems se encontró una diferencia estadística en el análisis post hoc.

Tabla No 3 Análisis del tamaño del efecto (ES), según características del ejercicio

\begin{tabular}{|c|c|c|c|c|c|}
\hline & ES & $\mathbf{D E}$ & $\mathbf{n}$ & $\mathbf{F}$ & Sig. \\
\hline Frecuencia de Sesiones & & & & 2.83 & $0.043^{*}$ \\
\hline a. 1 vez por semana ${ }^{a-c}$ & 0.27 & 0.19 & 8 & & \\
\hline b. $\quad 2$ veces por semana & 0.34 & 0.23 & 13 & & \\
\hline c. 3 veces por semana & 1.32 & 1.64 & 60 & & \\
\hline d. 4 o más veces por semana & 0.86 & 0.39 & 8 & & \\
\hline Duración de la Sesión & & & & 9.44 & $0.003^{*}$ \\
\hline a. De 30 a 35 minutos ${ }^{a-b}$ & 0.48 & 0.56 & 34 & & \\
\hline b. $\quad 60$ minutos & 1.39 & 1.66 & 55 & & \\
\hline Intensidad & & & & 1.57 & 0.202 \\
\hline a. Moderada & 0.64 & 0.19 & 10 & & \\
\hline b. De moderada a alta & 1.27 & 0.23 & 52 & & \\
\hline c. Ajustada por el paciente & 0.27 & 1.69 & 8 & & \\
\hline d. No especificado & 0.95 & 0.39 & 19 & & \\
\hline Total de Sesiones & & & & 7.86 & $0.001 *$ \\
\hline a. De 11 a $30^{\mathrm{a}-\mathrm{b}}$ & 0.45 & 0.39 & 36 & & \\
\hline b. De 31 a 50 & 1.57 & 1.79 & 46 & & \\
\hline c. Más de 51 & 0.58 & 0.21 & 7 & & \\
\hline Tamaño del Grupo & & & & 8.78 & $0.0001^{*}$ \\
\hline a. Menos de 10 personas ${ }^{\mathrm{a}-\mathrm{c}}$ & 0.45 & 0.40 & 12 & & \\
\hline b. De 11 a 20 personas & 0.54 & 0.34 & 37 & & \\
\hline c. De 21 a 30 personas & 1.68 & 1.90 & 40 & & \\
\hline
\end{tabular}

En la tabla $\mathrm{N}^{\circ} 4$ se muestran los resultados obtenidos luego de analizar el impacto del ejercicio acuático en los componentes de la salud física y mental que se ven alterados al padecer FM. Se destaca que indistintamente, todos los diferentes aspectos reciben un beneficio directo y significativo producto de la participación de un programa de ejercicio en un ambiente acuático. 
Tabla $N^{\circ} 4$ Análisis del tamaño del efecto (ES), según componentes de la salud física y mental para ejercicio acuático

\begin{tabular}{lccccc}
\hline & ES & DE & n & F & Sig. \\
\hline Dolor & & & & 1.76 & 0.065 \\
Fatiga & 0.97 & 0.91 & 18 & & \\
Puntos Sensibles & 2.78 & 3.25 & 5 & & \\
Calidad de Vida & 1.22 & 0.99 & 9 & & \\
Sueño & 0.79 & 0.62 & 3 & & \\
Depresión & 1.31 & 1.06 & 5 & & \\
Ansiedad & 0.77 & 0.73 & 11 & & \\
Impacto de la Enfermedad & 0.28 & 0.32 & 9 & & \\
Rigidez & 2.17 & 2.62 & 9 & & \\
Resistencia del Tren Inferior & 0.49 & 0.34 & 4 & & \\
Fuerza del Tren Inferior & 0.41 & 0.64 & 3 & & \\
Capacidad Cardiorrespiratoria & 0.47 & 0.34 & 4 & & \\
Resistencia del Tren Superior & 1.54 & 0.37 & 6 & & \\
\hline
\end{tabular}

\section{DISCUSIÓN}

El ejercicio acuático demostró su efectividad para enfrentar los síntomas experimentados al padecer FM. Este tipo de ejercicio fue el único que obtuvo diferencias significativas al ser comparado con el grupo control (ver tabla 1). Así mismo, el hecho de que la balneoterapia y el ejercicio acuático tuvieran los mayores promedios de tamaño de efecto, brinda información valiosa acerca de los beneficios del agua como ambiente curativo. Empero, la balneoterapia, al realizarse en aguas termales ("hot springs") la hace un tratamiento menos accesible para la mayoría de la población debido a la localización de las mismas, costos y la dependencia de un terapeuta, haciendo el ejercicio acuático un tratamiento más asequible.

Las propiedades del agua hacen que éste sea uno de los mejores ambientes para un programa de ejercicios en sujetos fibromiálgicos. Éste provee una resistencia natural, lo cual provoca una mejoría en el tono muscular, además la flotabilidad disminuye el impacto en las articulaciones. El ejercicio acuático mejora la flexibilidad y la fuerza en un ambiente que disminuye las molestias causadas por la enfermedad. Para una persona con este padecimiento es crucial tener los músculos saludables, eso por sí solo ofrece una sensación de alivio. Igualmente, la importancia de la fuerza muscular es vital para la realización de tareas diarias, ya que se facilita el movimiento (Silver, 2003).
Es importante recalcar los resultados positivos encontrados en este meta-análisis, ya que en todas las categorías de las variables moderadoras se dio un beneficio estadísticamente significativo en la sintomatología de la enfermedad. En todas las edades estudiadas, años de padecer la enfermedad, frecuencia del ejercicio, intensidades, tamaños de grupo, sesiones totales, síntomas y componentes de aptitud física se lograron mejorías significativas, lo que representa que el ejercicio acuático en esta enfermedad debe ser considerada una opción ideal a tomar en cuenta para su tratamiento.

Algunas características comunes en la metodología de los estudios desarrollados en esta área, limitaron posibles análisis en otras variables tales como el sexo, otros padecimientos asociados y la temperatura del agua. No obstante, los presentes hallazgos permiten establecer recomendaciones claras para las personas que padecen fibromialgia y establecer pautas para guiar futuras investigaciones en el área.

En cuanto a la edad de los pacientes, la mayoría de los estudios utilizaron pacientes con un promedio de 40 a 55 años. Esto se debe a que la prevalencia de la enfermedad se da en una edad avanzada, según una encuesta realizada a 6000 personas, el promedio de entrevistados que padecía de FM tenía 52.6 años (Chaitow, Baldry, Dommerholt \& Chambers, 2003). Por lo tanto, esto pudo influir en el hecho de que entre los grupos de 40 a 50 años y mayores de 50 años no hubiera diferencia en los beneficios del 
ejercicio acuático debido a que la mayoría de los enfermos tienen edades y características similares. Sin embargo, es importante mencionar que en ambos grupos etarios se lograron beneficios significativos.

La mejoría en la sintomatología se presenta independientemente del tiempo de manifestación de la enfermedad. No obstante, es importante tener en cuenta que la duración de los síntomas no necesariamente se relaciona con su severidad. Esta situación puede favorecer a que personas que han padecido la enfermedad durante muchos años y que tal vez no habían conseguido mejoras con otros tipos de terapias, logren una disminución en las molestias al realizar el ejercicio adecuado.

La frecuencia con la cual se realiza el ejercicio demostró ser una variable a ser tomada en cuenta al formular un programa de ejercicios en el agua dirigido a personas con FM. A mayor frecuencia en la realización de ejercicios hubo una tendencia a mayores tamaños de efecto. Sin embargo, la frecuencia de tres veces por semana generó los mejores resultados.

Aunque los estudios tienden a emplear duraciones similares en las sesiones de ejercicio. Las lecciones que mostraron mayores tamaños de efecto promedio para enfrentar la sintomatología de la enfermedad fueron aquéllas de 60 minutos. Sin embargo, no se debe descartar que a menores duraciones como de 30 a 35 minutos igualmente lograran aumentar el bienestar y el alivio en la sintomatología de estas personas

La tabla 3 muestra que, según el análisis de ANOVA, todos los pacientes, incluyendo aquellos que monitoreaban su propia intensidad de trabajo, experimentaron una mejoría en los síntomas de la FM independientemente de la intensidad a la que trabajaran. Los pacientes con FM tienen diferentes capacidades físicas iniciales. Algunos pacientes pueden ejercitarse a una intensidad moderada-alta, sin embargo, no la mayoría. Van Santen et al. (2002) compararon programas de intensidad baja y alta, encontrando que los pacientes se sienten adoloridos después de 24 horas de una sesión de entrenamiento de intensidad alta. Por lo mismo, la mayoría de estudios indican que el ejercicio de baja intensidad o intensidades ajustadas por el paciente son las mejor toleradas, ya que el paciente inicia un programa de manera cómoda, sin sentir dolor incapacitante y fatiga (Wallace \& Clauw, 2005).
Contrariamente, en este trabajo se observó un mayor tamaño de efecto promedio cuando la intensidad del ejercicio oscilaba entre moderada y alta, por lo tanto, se puede asumir que los pacientes que se ejercitan a intensidades más altas lograron mejores resultados. Sin embargo, es importante lograr un progreso paulatino en la intensidad que se utilizará para reducir el riesgo de deserción al programa de ejercicios.

Por otra parte, el tamaño del grupo es un factor situacional que afecta la motivación y adherencia en un programa de ejercicios. Aunque el tamaño de grupo ideal no se ha determinado de manera precisa, en este caso el efecto más positivo se observó en los tamaños de grupos de 20 a 30 personas en comparación a grupos con menos cantidad de personas. Este efecto se pudo deber a la motivación, ya que los pacientes se sienten identificados con sus compañeros al observar personas en su misma situación de salud. Así mismo, la empatía pudo jugar un papel importante, ya que, al identificarse con otras personas con los mismos padecimientos, se podría estar evitando el aislamiento y el abandono de la actividad, generados por la percepción de incomprensión al realizar el ejercicio en un grupo de personas saludables. Los lazos de amistad que pudieran formarse al estar en contacto con varios sujetos durante el periodo del ejercicio también pudo ser clave para lograr tan buenos resultados (Biddle, 2007).

Es importante tener en cuenta que si bien los mejores resultados se obtienen al participar en grupos grandes, también se presentan modificaciones positivas en la sintomatología cuando los grupos son pequeños. Esta situación sugiere que al realizar ejercicio en un ambiente acuático, las adaptaciones fisiológicas serían fundamentales para contribuir en la mejora de la sintomatología, no obstante, los aspectos psicosociales estarían potenciando estos cambios positivos.

A la luz de los resultados de este metaanálisis, se comprobó que el agua es uno de los mejores ambientes en los cuales las personas con FM pueden realizar ejercicios. Entre otras razones, esto podría deberse a que las personas pueden moverse con mayor facilidad y presentar un menor impacto en las articulaciones y músculos. Los beneficios en la calidad de vida de los pacientes, con la disminución de la sintomatología y mejoría de la aptitud física encontrados, demuestran que el ejercicio acuático 
debe ser considerado como una excelente opción de tratamiento de esta enfermedad.

En términos generales y a manera de conclusión, de acuerdo con la evidencia generada en este estudio, se recomienda realizar el ejercicio acuático fomentando los grupos grandes, que favorezcan los lazos sociales, ejecutando el programa con una frecuencia semanal de tres sesiones, con una duración de 60 minutos. Dado que a cualquier intensidad se pueden obtener modificaciones positivas, la misma no representa una variable moderadora de los beneficios obtenidos. No obstante, ante la gran cantidad de estudios que no reportaron la intensidad utilizada y la importancia de que la persona con fibromialgia permanezca en el programa de ejercicio, se recomienda establecer una intensidad de acuerdo a la capacidad de cada individuo.

\section{REFERENCIAS}

*Indica los artículos incluidos en el análisis estadístico meta-analítico.

*Altan, L., Bingöl, U., Aykaç, M., Koç, Z. \& Yurtkuran, M. (2004). Investigation of the effects of pool-based exercise on fibromyalgia syndrome. Rheumatology International, 24(5), 272-7.

*Assis, M.R., Silva, L.E., Alves, A.M., Pessanha, A.P., Valim, V., Feldman, D., Neto, T.L. \& Natour, J. (2006). A randomized controlled trial of deep water running: clinical effectiveness of aquatic exercise to treat fibromialgia. Arthritis and Rheumatism, 55(1), 57-65.

Biddle, S. (2007). Psychology of physical activity: determinants, well-being and interventions. Estados Unidos: Routledge.

Brecher, L.S. \& Cymet, T.C. (2004). A practical approach to fibromyalgia. Journal of the American Osteopathic Association, 101(4 Suppl Pt 2), S12-7. Review

Busch, A., Schachter, C.L., Peloso, P.M. \& Bombardier, C. (2002). Exercise for treating fibromyalgia syndrome. Cochrane Database System Review, 3, CD003786.

*Cedraschi, C., Desmeules, J., Rapiti, E., Baumgartner, E., Cohen, P., Finckh, A., Allaz, A.F. \& Vischer, T.L. (2004). Fibromyalgia: a randomised, controlled trial of a treatment programmed based on self management. Annals of Rheumatic Diseases, 63(3), 290-296.

Chakrabarty, S.\& Zoorob, R. (2007). Fibromyalgia. American Family Physician, 76(2), 247-54.

Chaitow, L, Baldry, P., Dommerholt, J. y Chambers, G. (2003). Fibromyalgia syndrome: $a$ practitioner's guide to treatment. Estados Unidos: Elsevier Health Sciences.

Clauw, D.J. \& Crofford, L.J. (2003). Chronic widespread pain and fibromyalgia: what we know, and what we need to know. Best Practice Research: Clinical Rheumatology, 17(4), 685-701.

*De Andrade, S. De Carvalho, R., Soares, A., De Abreu, R., De Madeiros, L., \& Vilar, M. (2008). Thalassotherapy for fibromyalgia: a randomized controlled trial comparing aquatic exercises in sea water and water pool. Rheumatology International, 29(2), 147-52.

De Melo, D., de Carvalho, L.\& do Prado, G. (2006). Hydrotherapy and conventional psysiotherapy improve total sleep time and quality of life of fibromyalgia patients. Sleep Medicine 7, 293296.

*Dos Santos, L., Pastore, C., Yazbek, P., Harumi, M., Gonçalves, A., Seguchi, H., \& Rizzo L., (2007). Effects of physical conditioning over patients with fibromyalgia. Rev. Bras Med Esporte, 13(1), 5-8.

*Evcik, D., Yigit, I., Pusak, H. \& Kavuncu, V. (2008). Effectiveness of aquatic therapy in the treatment of fibromyalgia syndrome: A randomized controlled open study. Rheumatology International, 28(9), 885-90

Gowans, S.E., DeHueck, A .\& Abbey, S.E. (2002). Measuring exercise-induced mood changes in fibromyalgia: a comparison of several measures. Arthritis Rheumatology, 47(6), 6039.

*Gowans, S.E., deHueck, A., Voss, S. \& Richardson, M. (1999). A randomized, controlled trial of exercise and education for individuals with fibromyalgia. Arthritis Care and Research, 12(2), 120-8.

*Gowans, S.E., deHueck, A., Voss, S., Silaj, A., Abbey, S.E. \& Reynolds, W.J. (2001). Effect of a randomized, controlled trial of exercise on mood and physical function in individuals with fibromyalgia. Arthritis Rheumatology, 45(6), 519-29. 
*Gusi, N., Tomas-Carus, P., Häkkinen, A., Häkkinen, K. \& Ortega-Alonso, A. (2006). Exercise in waist-high warm water decreases pain and improves health-related quality of life and strength in the lower extremities in women with fibromyalgia. Arthritis Rheumatology, 55(1), 66-73.

Hassett, A.L., Cone, J.D., Patella, S.J. \& Sigal, L.H. (2000). The role of catastrophizing in the pain and depression of women with fibromyalgia syndrome. Arthritis Rheumatology, 43(11), 2493-500.

Hinman, R.S., Heywood, S.E. \& Day, A.R. (2007). Aquatic physical therapy for hip and knee osteoarthritis: results of a single-blind randomized controlled trial. Physical Therapy, 87(1), 32-43.

*Jentoft, E.S., Kvalvik, A.G. \& Mengshoel, A.M. (2001). Effects of pool-based and land-based aerobic exercise on women with fibromyalgia/chronic widespread muscle pain. Arthritis Rheumatology, 45(1), 42-7.

*Mannerkorpi, K., Nyberg, B., Ahlmén, M. \& Ekdahl, C. (2000). Pool exercise combined with an education program for patients with fibromyalgia syndrome. A prospective, randomized study. Journal of Rheumatology, 27(10), 2473-81.

McBeth, J., Macfarlane, G.J., Benjamin, S. \& Silman AJ. (2001). Features of somatization predict the onset of chronic widespread pain: results of a large population-based study. Arthritis Rheumatology, 44(4), 940-6.

Minor, M., Hewett, J., Webel, R., Anderson, S., \& Kay, D. (1989). Efficacy of physical conditioning exercise in patients with rheumatoid arthritis and osteoarthritis. Arthritis Rheumatology, 32(11), 1396-1405.

*Munguía-Izquierdo, D. \& Legaz-Arrese, A. (2007). Exercise in warm water decreases pain and improves cognitive function in middle-aged women with fibromyalgia. Clinical and Experimental Rheumatology, 25(6), 823-30.

Peterson, E.L. (2007). Fibromyalgia--management of a misunderstood disorder. Journal of the American Academy of Nurse Practitioners, 19(7), 341-8.

*Redondo, J.R., Justo, C.M., Moraleda, F.V., Velayos, Y.G., Puche, J.J., Zubero, J.R., Hernández, T.G., Ortells, L.C. \& Pareja, M.A. (2004). Long-term efficacy of therapy in patients with fibromyalgia: a physical exercisebased program and a cognitive-behavioral approach. Arthritis and Rheumatism, 51(2), 184-92.

*Ritomy, M., Laurindo, M., Rodríguez, A. \& Tanaka, C. (2008). Effect of Aquatic Respiratory Exercise Based Program in Patients with Fibromyalgia. International Journal of Rheumatic Diseases, 11, 131-140.

Rooks, D.S., Silverman, C.B. \& Kantrowitz, F.G. (2002). The effects of progressive strength training and aerobic exercise on muscle strength and cardiovascular fitness in women with fibromyalgia: a pilot study. Arthritis Rheumatology, 47(1), 22-8.

Rosas, R. (2006). Fibromialgia: etiología, clínica, diagnóstico y tratamiento. Offarm, 25, 94-100.

Silver, D. (2003). Playing Through Arthritis: How to Conquer Pain and Enjoy Your Favorite Sports and Activities. Estados Unidos: McGraw-Hill Professional.

Thomas, J., \& French, K. (1986). The use of metaanalysis in exercise and sport: A tutorial. Research Quarterly for Exercise and Sport. 57, (3), 196-204.

Thomas, R., Salazar, W. \& Landers, D. (1991). What is missing in $\mathrm{p}<.05$ ? Effect size. Research Quarterly for Exercise and Sport, 62(3), 344-348.

*Tomas-Carus, P, Häkkinen, A, Gusi, N, Leal, A, Häkkinen, K \& Ortega-Alonso, A. (2007). Aquatic training and detraining on fitness and quality of life in fibromyalgia. Medicine and Science in Sports and Exercise, 39 (7), 104450.

*Tomas-Carus, P., Gusi, N., Häkkinen, A., Häkkinen, K., Leal, A., \& Ortega, A. (2008). Eight months of physical training in warm water improves physical and mental health in women with fibromyalgia: a randomized controlled trial. Journal of Rehabilitation Medicine, 40, 248-252.

Van Santen M, Bolwijn P, Landewe R, Verstappen F, Bakker C, Hidding A, van der Heijde D, Houben H \& van der Linden S. (2002) High or low intensity aerobic fitness training in fibromyalgia: Does it matter? $J$ Rheumatol, 29:582-587

Wallace, D. \& Clauw, D. (2005). Fibromyalgia \& other central pain syndromes. Estados Unidos: Lippincott Williams \& Wilkins 
Wang, T.J., Belza, B., Thompson, F., Whitney, J.D. \& Bennett K. (2007). Effects of aquatic exercise on flexibility, strength and aerobic fitness in adults with osteoarthritis of the hip or knee. Journal of Advanced Nursing, 57(2), 141-52 (ABSTRACT)

Wolfe, F., Ross, K., Anderson, J., Russell, I.J. \& Hebert, L. (1995) The prevalence and characteristics of fibromyalgia in the general population. Arthritis and Rheumatism, 38(1), 19-28.

Participación: A- Financiamiento B- Diseño del estudio CRecolección de datos D- Análisis estadístico e interpretación de resultados E- Preparación de manuscrito. 